\title{
Photosynthesis by Isolated Chloroplasts
}

\section{REVERSAL OF ORTHOPHOSPHATE INHIBITION BY CALVIN-CYCLE INTERMEDIATES}

\author{
BY W. COCKBURN, D. A. WALKER AND C. W. BALDRY \\ Department of Botany, Imperial College, London, S.W. 7
}

(Received 22 September 1967)

\begin{abstract}
1. The orthophosphate inhibition of photosynthesis by isolated spinach chloroplasts can be reversed by 3-phosphoglycerate, dihydroxyacetone phosphate, glyceraldehyde 3-phosphate, fructose 6-phosphate and fructose 1,6-diphosphate. 2. Metabolically related compounds such as ribulose 1,5-diphosphate, glucose 6-phosphate, 6-phosphogluconate and phosphoenolpyruvate are ineffective. 3. The kinetics of reversal are characteristic of the intermediate used, but, in each instance, the onset of oxygen evolution is accompanied by a carbon dioxide fixation and except with 3-phosphoglycerate the stoicheiometry is close to unity. 4. The nature of orthophosphate inhibition and its reversal is discussed in relation to metabolic control of photosynthesis.
\end{abstract}

Isolated chloroplasts with intact envelopes wilh assimilate carbon dioxide and evolve oxygen (see, e.g., Gibbs, 1967). Maximum rates are not achieved immediately but only after an initial lag (Gibbs \& Bamberger, 1962; Bamberger \& Gibbs, 1963; Walker, 1965a), which varies with experimental conditions (Walker, 1965b; Gibbs, Bamberger, Ellyard \& Everson, 1965; Bucke, Walker \& Baldry, 1966; Baldry, Walker \& Bucke, 1966b; Baldry, Bucke \& Walker, 1966a). If the concentration of orthophosphate in the reaction mixtures is raised above about $0.05 \mathrm{~mm}$, the induction period is prolonged and the maximum rate is decreased (Cockburn, Baldry \& Walker, 1967a). Further increases in orthophosphate concentration bring about further extensions of the lag and corresponding decreases in rate (Cockburn, Baldry \& Walker, 1967c; Kalberer, Buchanan \& Arnon, 1967). A concentration of $6.6 \mathrm{~mm}$-orthophosphate is usually sufficient to suppress the oxygen evolution associated with carbon dioxide assimilation almost entirely. Results given below show that this inhibition may be reversed by the addition of any of several Calvin-cycle intermediates (Bassham \& Calvin, 1957).

\section{MATERIALS AND METHODS}

Spinach was generously given by Mr W. Mackenzie and his colleagues at the Chelsea Physic Garden or bought on the local market (Covent Garden).

Chloroplasts. Spinach leaf $(50 \mathrm{~g}$.) was disrupted by 3-5 sec. maceration in a motor-driven domestic food blender containing $200 \mathrm{ml}$. of grinding medium that had previously been chilled (by agitation in a cold bath) to the consistency of melting snow. The juice was squeezed from the pulp manually through two layers of muslin and filtered through a further eight layers. The chloroplasts were then rapidly separated from the medium in $50 \mathrm{ml}$. tubes at $0^{\circ}$ in an angle-head centrifuge that was taken from rest to approx. $4000 \mathrm{~g}$ and back to rest in approx. 60 sec. The supernatant was decanted and the pellets resuspended, by using cotton wool and a glass rod, in $1.0 \mathrm{ml}$. of cold suspending medium. The entire preparation was carried out as quickly as possible (about $5 \mathrm{~min}$.).

Grinding medium. This was sorbitol $(0 \cdot 33 \mathrm{M})$ containing $\mathrm{Na}_{4} \mathrm{P}_{2} \mathrm{O}_{7}(0.01 \mathrm{M})$ and $\mathrm{MgCl}_{2}(0.1 \%, \mathrm{w} / \mathrm{v})$ adjusted to pH 6.5 with $\mathrm{HCl}$.

Suspending medium. This was sorbitol $(0 \cdot 33 \mathrm{M})$ containing $0.05 \mathrm{M}-N$-2-hydroxyethylpiperazine- $N^{\prime}$-2-ethanesulphonic acid (Good et al. 1966), $\mathrm{MgCl}_{2}$ (1 $\mathrm{mm}$ ), $\mathrm{MnCl}_{2}$ (1 $\mathrm{mM}$ ) and EDTA (2mM) adjusted to $\mathrm{pH} 7 \cdot 6$ with $\mathrm{NaOH}$.

Reaction mixtures. The chlorophyll content (Arnon, 1949) was adjusted to a constant quantity $(100 \mu \mathrm{g}$.). Water and double-strength suspending medium were used in appropriate volumes so that reaction mixtures (volume $1 \mathrm{ml}$.) finally contained the components of the suspending medium in unchanged concentration. Carbon dioxide was usually provided as $\mathrm{NaHCO}_{3}(10 \mu$ moles $)$, but in experiments with radioactive $\mathrm{CO}_{2}$ it was found more convenient to use $\mathrm{NaH}^{14} \mathrm{CO}_{3}(400 \mu \mathrm{c}, 7 \cdot 5 \mu$ moles $)$ at a slightly lower concentration. Calvin-cycle intermediates were added from microsyringes in small volumes $(20 \mu \mathrm{l}$.) containing $2 \mu$ moles. All solutions other than the bicarbonate were used at approximately the same $\mathrm{pH}(\mathbf{7 \cdot 6 )}$ as the suspending medium. Oxygen and carbon dioxide were measured simultaneously in the same vessel.

Measurement of carbon dioxide. Samples $(10 \mu \mathrm{l}$.) were withdrawn from reaction mixtures (with a micro-syringe) at the times indicated (Figs. 6 and 7) and injected into individual tubes containing $40 \mu \mathrm{l}$. of $\mathrm{HCl}$ (approx. $0 \cdot 3 \mathrm{~N}$ ). Samples $(20 \mu$ l.) were transferred to uniform lens-tissue disks on aluminium planchets and dried briefly under an infrared 
lamp. Radioactivity was determined with a NuclearChicago gas-flow counter (model D47).

Calibration. The values of the specific activity and radioativity of the $\mathrm{NaH}^{14} \mathrm{CO}_{3}$ provided by The Radiochemical Centre (Amersham, Bucks.) were accepted without further verification. The radioactivity of a solution containing [ $\left.{ }^{14} \mathrm{C}\right]$ sucrose (The Radiochemical Centre) was checked against standards in a scintillation counter. Small quantities of this solution were added to reaction mixtures containing chloroplasts that were then sampled and measured as described above. This procedure, which eliminates errors resulting from variable geometry and self-absorption, was used as a basis for equating counts/min. recorded with carbon dioxide converted into an acid-stable form.

Measurement of oxygen. Polarographic measurements (platinum-silver-silver chloride) were made in specially constructed cylindrical Perspex vessels $(1 \mathrm{~cm} . \times 5 \mathrm{~cm}$.) based on an original design by Professor Brian Chappell (University of Bristol). The platinum electrode was situated on the floor of the cell from which it was separated by a Teflon membrane. The contents were stirred rapidly (magnetic stirrer and glass-covered follower). Each vessel was closed by a cylindrical plunger, communicating with the exterior by a central capillary (for sampling etc.). Water (at $19-20^{\circ}$ ) from a thermostatically controlled bath was pumped through an outer jacket at 4-51./min. The electrode currents from two such vessels were used to create small voltages that were recorded simultaneously and continuously on a two-pen recorder (Rikandenki, model B241). Experiments were carried out aerobically. The oxygen in solution at the onset of measurement was approx. $0.28 \mu \mathrm{mole} / \mathrm{ml}$.

Calibration. The base line was set by the addition of small quantities of $\mathrm{Na}_{2} \mathrm{~S}_{2} \mathrm{O}_{4}$ and the air line by air-saturated water at $20^{\circ}$. Additional checks on linearity were carried out by injecting small quantities of dilute (standardized) $\mathrm{H}_{2} \mathrm{O}_{2}$ solution into suspending medium to which catalase had been added.

Illumination. Each reaction vessel was illuminated by a quartz-iodine slide-projector (150w, 24v). Additional focusing was provided by a spherical flask containing water $(15 \mathrm{~cm}$. light-path) that also served as a heat filter. The beam was then passed through a red Perspex filter $(80 \%$ transmission above $600 \mathrm{~m} \mu$ ) and a Balzer heat filter (Calflex C, Tempax). Light passing through the vessel was also reflected back into the suspension from a semi-cylindrical aluminium mirror.

Equilibration. Reaction mixtures were allowed to equilibrate at $20^{\circ}$ for $3 \mathrm{~min}$. in the dark before the addition of a small volume of chloroplast suspension $30 \mathrm{sec}$. before illumination.

\section{RESULTS}

Inhibition of oxygen evolution by orthophosphate. Fig. 1. shows the degree of suppression of oxygen evolution by orthophosphate in a typical preparation of intact chloroplasts from spinach leaves. In chloroplasts prepared immediately from freshly gathered illuminated leaves the degree of inhibition may be less, presumably because of the persistence of Calvin-cycle intermediates (see below). Once separated from the parent tissue the photosynthetic

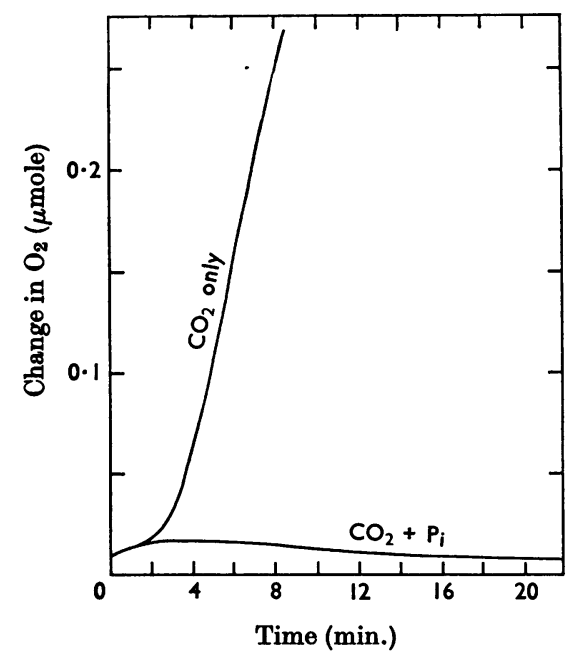

Fig. 1. Orthophosphate inhibition of $\mathrm{O}_{2}$ evolution by intact spinach chloroplasts with $\mathrm{CO}_{2}$ as the sole added substrate. Both reaction mixtures (see the Materials and Methods section) contained bicarbonate. In addition one contained $10 \mu$ moles of orthophosphate.

activity of isolated chloroplasts rarely remains constant for long periods even when kept at $0^{\circ}$ in the dark. In this and other experiments (below) oxygen was therefore measured simultaneously in two mixtures containing chloroplasts from the same preparation. For example in Fig. 1. orthophosphate was added to one reaction mixture and not to the other and the recordings of oxygen evolution were made concurrently rather than consecutively.

Reversal of orthophosphate inhibition by ribose 5-phosphate and dihydroxyacetone phosphate. Fig. 2 (a) shows that the addition of dihydroxyacetone phosphate to chloroplasts illuminated in a mixture containing an inhibitory concentration $(10 \mathrm{~mm})$ of orthophosphate was followed almost immediately by a rapid oxygen evolution. This is compared with the reversal initiated by the addition of ribose 5-phosphate at the same moment to a similar reaction mixture. The same degree of reversal was again achieved, but the maximal rate of evolution was only reached after some minutes.

Reversal by 3-phosphoglycerate and by fructose 1,6-diphosphate. Fig. 2(b) shows that 3-phosphoglycerate reversed the orthophosphate inhibition even more readily than dihydroxyacetone phosphate (cf. Figs. $2 a$ and $2 b$ ), whereas fructose 1,6diphosphate took longer to exert its effect than ribose 5-phosphate.

Reversal by fructose 6-phosphate. An even longer period elapsed (Fig. 3) between the addition of fructose 6-phosphate and attainment of the 


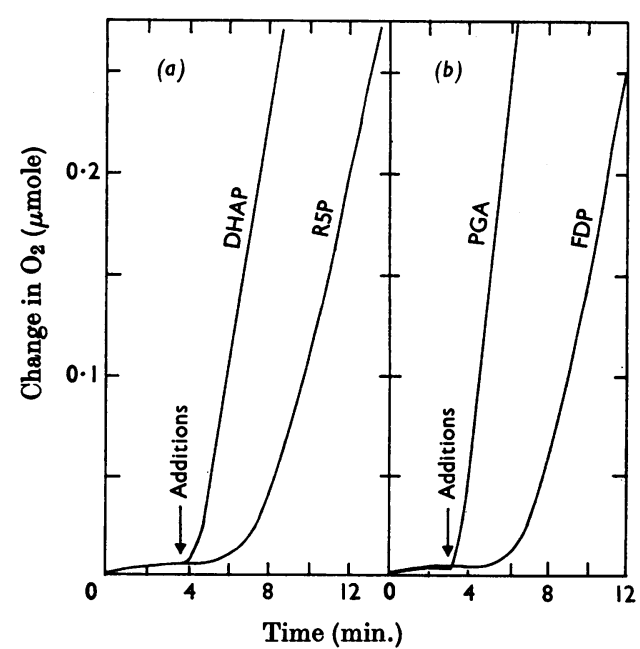

Fig. 2. Reversal of orthophosphate inhibition. All reaction mixtures (see the Materials and Methods section) contained bicarbonate and an 'inhibitory' amount (10 $\mu$ moles) of orthophosphate. Dihydroxyacetone phosphate (DHAP), ribose 5-phosphate (R5P), 3-phosphoglycerate (PGA) and fructose 1,6-diphosphate (FDP) ( $2 \mu$ moles of each) were added as indicated. It should be noted that with ribose 5-phosphate and fructose 1,6-diphosphate there was a delay of some minutes before the maximum rate was achieved.

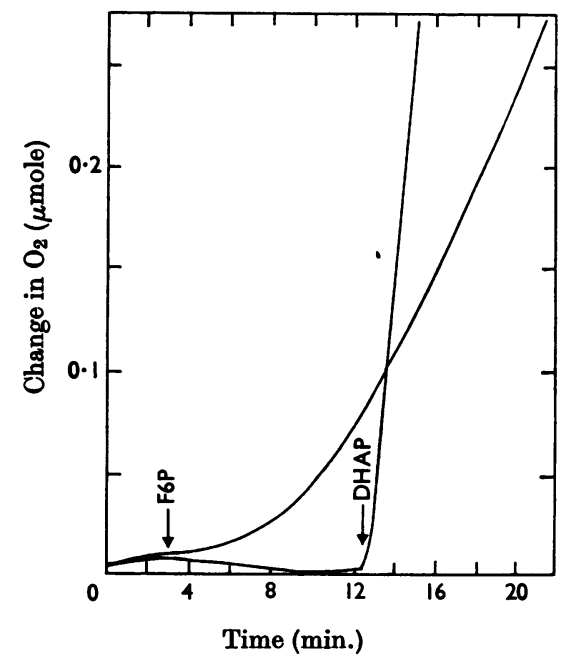

Fig. 3. Reversal of orthophosphate inhibition. Both reaction mixtures (see the Materials and Methods section) contained bicarbonate and an 'inhibitory' amount (10 $\mu$ moles) of orthophosphate. The addition of fructose 6-phosphate (F6P) ( $2 \mu$ moles) was followed by a very gradual increase in $\mathrm{O}_{2}$ evolution, which contrasts with the very rapid $\mathrm{O}_{2}$ evolution after the later addition of dihydroxyacetone phosphate $(2 \mu \mathrm{moles})$ to the control. maximum rate. In this experiment the control (which also contained $10 \mathrm{~mm}$-orthophosphate) was allowed to run for just over $12 \mathrm{~min}$. without additions. At this point the addition of dihydroxy. acetone phosphate again brought about a very rapid reversal, which contrasts sharply with the slow increase in oxygen production that followed the addition of fructose 6-phosphate.

Reversal by glyceraldehyde 3-phosphate. The response to glyceraldehyde 3-phosphate was similar to that produced by dihydroxyacetone phosphate (cf. Figs. $2 a, 3$ and $4 b$ ).

Related metabolites that failed to reverse the orthophosphate inhibition. Fig. 4(a) shows that glucose 6-phosphate, unlike fructose 6-phosphate, failed to produce any measurable response during the period of measurement. In this and similar experiments both reaction mixtures contained $10 \mathrm{~mm}$-orthophosphate and one was allowed to act as a control for some minutes after the addition of a metabolite to the other. Reversal was then demonstrated in both mixtures by the addition of 3-phosphoglycerate (Fig. 4a), glyceraldehyde 3-phosphate (Fig. $4 b$ ) or dihydroxyacetone phosphate. Fig. 4(b) shows that 6-phosphogluconate was as ineffective as glucose 6-phosphate and failed to bring about reversal. Other related metabolites that failed to produce a detectable response included ribulose 1,5-diphosphate, phosphoenolpyruvate and free ribose (cf. Bucke et al. 1966; Baldry et al. 1966b).

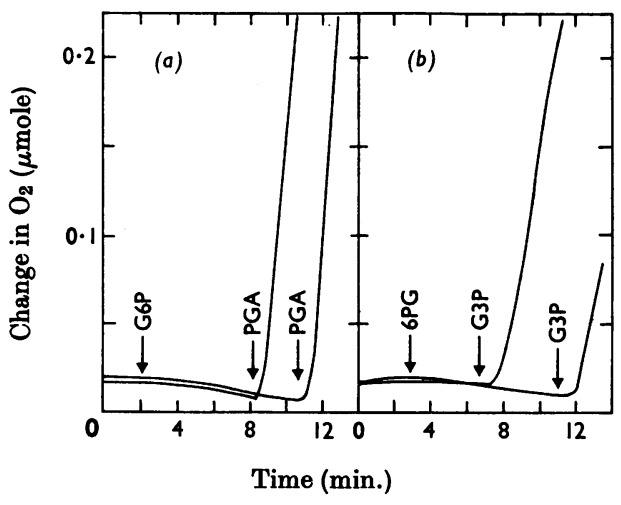

Fig. 4. Failure of glucose 6-phosphate and 6-phosphogluconate to reverse orthophosphate inhibition. All reaction mixtures (see the Materials and Methods section) contained bicarbonate and an 'inhibitory' amount ( $10 \mu \mathrm{moles})$ of orthophosphate. Glucose 6-phosphate (G6P) and 6-phosphogluconate (6PG) ( $2 \mu$ moles of each) were added as indicated to one of each pair of reaction mixtures, while the other was left as a control. Reversal was subsequently demonstrated in all mixtures by the addition of 3-phosphoglycerate (PGA) (2 $\mu$ moles) or glyceraldehyde 3-phosphate (G3P) ( $2 \mu$ moles) as indicated. 


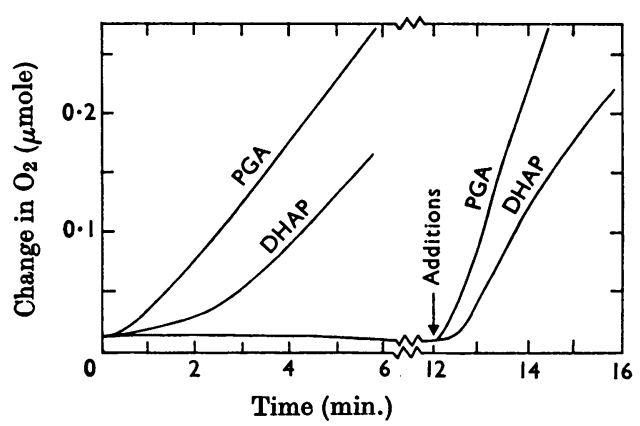

Fig. 5. Effect of pre-illumination on the kinetics of $\mathrm{O}_{2}$ evolution in the presence of 3-phosphoglycerate or dihydroxyacetone phosphate. All four reaction mixtures (see the Materials and Methods section) contained bicarbonate and an 'inhibitory' amount (10 $\mu$ moles) of orthophosphate. Dihydroxyacetone phosphate (DHAP) ( $2 \mu$ moles) or 3-phosphoglycerate (PGA) ( $2 \mu$ moles) was added as indicated in the dark before illumination or after approx. $12 \mathrm{~min}$. in the light. It should be noted that with dihydroxyacetone phosphate pre-illumination decreased the time taken to attain the maximum rate from more than $3 \mathrm{~min}$. to about $45 \mathrm{sec}$.

Relationship between the duration of illumination and the rapidity of response. A close comparison of Figs. 2(a) and 3 suggests that the response to dihydroxyacetone phosphate becomes more rapid as the time that elapses before its addition is prolonged. This is seen more clearly in Fig. 5, in which recordings were made at the maximum available speed of $20 \mathrm{~mm}$. $/ \mathrm{min}$. In Fig. 5, dihydroxyacetone phosphate was added to one mixture and 3-phosphoglycerate to the other, immediately before illumination, and also after 12 min. illumination. With both additives, the effect of pre-illumination was to increase the maximum rate and to shorten the lag. However, the effect was more marked with dihydroxyacetone phosphate, which, after pre-illumination, produced a response closer to that of 3-phosphoglycerate, in the sense that the time that elapsed before attainment of the maximum rate was decreased from more than $3 \mathrm{~min}$. to less than $1 \mathrm{~min}$. It should be noted that these responses were brought about in mixtures containing $10 \mathrm{~mm}$-orthophosphate. Under other conditions the onset of oxygen evolution in the presence of 3-phosphoglycerate may be even more rapid (e.g. see Walker \& Hill, 1967).

Simultaneous measurements of oxygen evolution and carbon dioxide fixation. The oxygen evolution observed in the presence of substrate concentrations of 3-phosphoglycerate (Walker \& Hill, 1967) is not initially dependent on the presence of carbon dioxide (Walker, Cockburn \& Baldry, 1967),

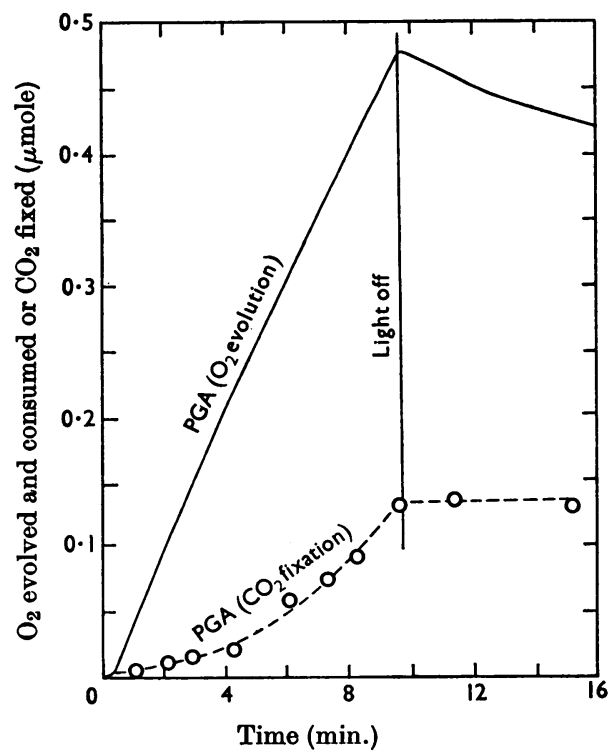

Fig. 6. Simultaneous measurements of $\mathrm{O}_{2}$ evolution (-) and ${ }^{14} \mathrm{CO}_{2}$ fixation (---) in the presence of 3-phosphoglycerate and an 'inhibitory' amount of orthophosphate. The single reaction mixture contained $\mathrm{NaH}^{14} \mathrm{CO}_{3}$ (see the Materials and Methods section), 3-phosphoglycerate

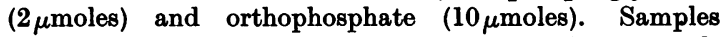
$(10 \mu l$.) for radioactivity measurements (see the Materials and Methods section) were removed at the times indicated and immediately injected into $0 \cdot 3 \mathrm{~N}-\mathrm{HCl}(40 \mu \mathrm{l}$.). It will be seen that $\mathrm{O}_{2}$ evolution started almost immediately whereas fixation increased gradually. When illumination was stopped (after approx. $10 \mathrm{~min}$.) $\mathrm{O}_{2}$ evolution was replaced by $\mathrm{O}_{2}$ uptake.

presumably because 3-phosphoglycerate can undergo direct conversiơn into 1,3-diphosphoglycerate, which then serves as the hydrogen acceptor. In mixtures containing both carbon dioxide and 3-phosphoglycerate oxygen evolution started almost immediately, but, not surprisingly, carbon dioxide fixation began slowly and accelerated gradually over a period of some minutes (Fig. 6). However, in the presence of sufficient orthophosphate to inhibit carbon dioxide fixation, when the addition of 3-phosphoglycerate was delayed for some minutes as before (cf. Figs. $2 b, 4 a$ and 5), the result was different. Again oxygen evolution started promptly and reached its maximum rate within seconds of the addition of 3-phosphoglycerate, but this time it was closely followed by a qualitatively similar fixation of carbon dioxide (Fig. 7a). With dihydroxyacetone phosphate (Fig. $7 b$ ) added simultaneously to the second electrode vessel the results were much the same except that the values for carbon dioxide fixation agreed even more closely 


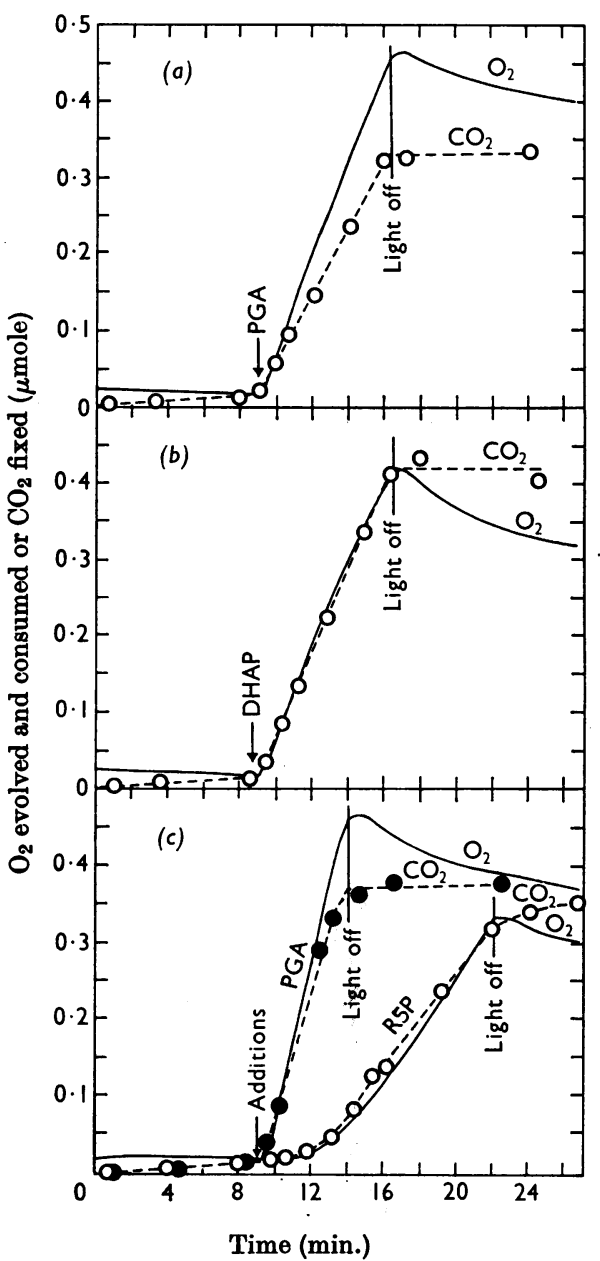

Fig. 7. Simultaneous measurements of $\mathrm{O}_{2}$ evolution and consumption (-) and ${ }^{14} \mathrm{CO}_{2}$ fixation (---) during reversal of orthophosphate inhibition by 3-phosphoglycerate (PGA), dihydroxyacetone phosphate (DHAP) and ribose 5-phosphate (R5P). Each reaction mixture contained an 'inhibitory' amount (10 $\mu$ moles) of orthophosphate and $\mathrm{NaH}^{14} \mathrm{CO}_{3}$ (see the Materials and Methods section). Records illustrated in $(a)$ and $(b)$ were obtained simultaneously with the same chloroplast preparation. Dihydroxyacetone phosphate ( $2 \mu$ moles) and 3-phosphoglycerate ( $2 \mu$ moles) were added to separate reaction mixtures at the same moment, so that $(a)$ and $(b)$ may be directly compared. The same procedure was used in (c), again with the same chloroplast preparation; (c) illustrates the changes after the addition of ribose 5-phosphate ( $2 \mu$ moles). 3-Phosphoglycerate ( $2 \mu$ moles) was added to a separate identical reaction mixture at the same time and the recorded values are also included in (c) to permit direct comparison. It should be noted that in each case the progress curves for $\mathrm{O}_{2}$ and $\mathrm{CO}_{2}$ are closely associated (in the light) and that with ribose 5-phosphate and dihydroxyacetone phosphate the ratio of $\mathrm{CO}_{2}$ fixed to $\mathrm{O}_{2}$ evolved was close to unity. with the oxygen-production trace. With ribose 5-phosphate the characteristically slow response of oxygen evolution was matched by a correspondingly slow onset of carbon dioxide assimilation (Fig. 7c), and the kinetics of oxygen evolution were markedly different from those obtained when 3-phosphoglycerate was added simultaneously to the second vessel for comparison. Again it will be noted (cf. Figs. $7 a$ and $7 c$ ) that there was a greater discrepancy between oxygen evolution and carbon dioxide assimilation with 3-phosphoglycerate than with the other additives and that this probably represents a genuine excess of oxygen as in Fig. 6.

Post-illumination oxygen uptake. In previous experiments (Walker \& Hill, 1967), in which carbon dioxide fixation and oxygen evolution were measured simultaneously, oxygen uptake in the dark was considerable (largely because of autoxidation of the suspending medium, which contained GSH and ascorbate). To arrive at an estimate of the stoicheiometry between carbon dioxide fixed and oxygen evolved it was therefore thought necessary to make an appropriate correction for both the initial uptake in the dark and an increased uptake after illumination. In the present experiments an improved procedure was used for the separation of chloroplasts (see the Materials and Methods section) and the mixtures contained no added ascorbate or GSH. Oxygen uptake in the dark was only a small fraction of oxygen production in the light. However, we have consistently observed immediately after illumination an increased oxygen uptake that slowly decreases until it returns to that measured in the dark before illumination (Figs. 6 and 7). Whether the post-illumination oxygen uptake value is added to the net oxygen evolution or disregarded it will be seen that (except with 3-phosphoglycerate) the stoicheiometry remains close to $1 \mathrm{~mol}$. of oxygen produced $/ \mathrm{mol}$. of carbon dioxide fixed. Conversely, if a stoicheiometry of $1: 1$ is accepted it would be difficult to conclude that the post-illumination oxygen uptake reflected enhanced uptake in the light rather than dark reoxidation of some readily oxidized compound (perhaps endogenous ascorbate) that is held in a reduced state during photosynthesis. In a dozen or more experiments carried out under various conditions we have found no indication that the post-illumination oxygen uptake is associated with any release of ${ }^{14} \mathrm{CO}_{2}$ (cf. Walker \& Hill, 1967).

\section{DISCUSSION}

The nature of the orthophosphate inhibition of photosynthesis by isolated chloroplasts (e.g. see Cockburn et al. 1967a,b,c; Kalberer et al. 1967) is uncertain. Orthophosphate is a known (Weissbach, Horecker \& Hurwitz, 1956) competitive (Paulsen \& 
Lane, 1966) inhibitor of ribulose 1,5-diphosphate carboxylase. If orthophosphate acts by inhibiting carboxylation alone, then the oxygen evolution seen in the presence of substrate concentrations of 3-phosphoglycerate should be insensitive to the inhibitor since this reaction system is, at the outset, entirely or largely independent of carbon dioxide (Walker et al. 1967). In fact such lack of sensitivity has been reported (Cockburn et al. 1967b). It was therefore not surprising that the addition of 3-phosphoglycerate (to illuminated mixtures containing chloroplasts, carbon dioxide and an inhibitory concentration of orthophosphate) was followed by an almost immediate evolution of oxygen (Figs. $2 b, 4 a, 5$ and $7 a$ ). What was unexpected (Figs. $7 a$ and $7 c$ ) was that this oxygen evolution should be associated with a fixation of carbon dioxide that started with equal rapidity. The situation here is clearly different from that in which 3-phosphoglycerate is added (with carbon dioxide) in the dark. The oxygen evolution that then starts almost immediately on illumination is not closely followed by fixation, which by contrast begins slowly and builds up in the next few minutes (Fig. 6). It indicates that 3-phosphoglycerate acts as it does, not only because it is independent of carboxylation, but also by direct or indirect reversal of the orthophosphate inhibition. This is supported by the similar results obtained with dihydroxyacetone phosphate and glyceraldehyde 3-phosphate, the products of 3-phosphoglycerate reduction. These compounds cannot bring about oxygen evolution in the absence of carbon dioxide (Walker et al. 1967) and it must be assumed that they contribute to the production of the hydrogen acceptor (1,3-diphosphoglycerate) via carboxylation and the reactions of the Calvin cycle (Bassham \& Calvin, 1957). Thus if 3-phosphoglycerate can initiate carbon dioxide fixation (Fig. 7a) and dihydroxyacetone phosphate can bring about oxygen evolution (Fig. 7b) the intervening reactions embrace the entire Calvin cycle.

All the compounds that effected reversal have been shown to stimulate carbon dioxide fixation, in substrate or catalytic quantities, when added to chloroplasts separated in orthophosphate buffers (Bucke et al. 1966; Baldry et al. 1966b). The difference in response time between the triose phosphates, pentose phosphates and hexose phosphates may reflect differences in their ability to penetrate the intact chloroplast envelope (and indeed preincubation in the dark may decrease the lag that, for example, follows the addition of fructose 1,6-diphosphate), but it is more likely that the additional time is consumed in bringing about metabolic changes. It may be noted that Gibbs et al. (1965) regard glyceraldehyde 3-phosphate as a possible 'pacemaker' in the reductive pentose phosphate pathway and that all of the effective intermediates are readily converted into this triose phosphate by known routes. Ribulose 1,5-diphosphate was ineffective in bringing about reversal, but we have found that, to procure the best rate of dark carbon dioxide fixation with ribulose 1,5diphosphate as substrate, it is necessary to rupture the chloroplast envelope, and it seems probable that ribulose diphosphate does not readily enter the intact chloroplast (cf. Jensen \& Bassham, 1966; Heber, 1965).

The actual mechanism of reversal is still a matter of speculation. However, two enzymes of the cycle, ribose 5-phosphate isomerase (Anderson, Worthen \& Fuller, 1967) and ribulose 1,5-diphosphate carboxylase (Paulsen \& Lane, 1966), are sensitive to orthophosphate and the latter is known to be inhibited in a competitive manner. By its very nature, a competitive inhibition will tend to be reversed if the concentration of substrate is increased. The rapidity of reversal would then be related to the speed at which the substrate was increased and to the relative affinities of the enzymes for their substrates and for the inhibitor, orthophosphate. Clearly any intermediate that could feed into the Calvin cycle would contribute to reversal and the kinetics would reflect the ease of penetration of the chloroplast envelope and the extent of their subsequent metabolism. It may therefore not be necessary to look beyond this explanation, although, in view of the growing body of facts relating to allosteric inhibition and activation, a more subtle interaction cannot be discounted. However, in preliminary experiments with ribulose 1,5-diphosphate carboxylase we have found no indication that the orthophosphate inhibition is overcome by any of the intermediates that bring about reversal in the intact chloroplast.

Finally, we are impressed by the extent to which carbon dioxide fixation and oxygen production are linked. Only in experiments in which 3-phosphoglycerate is added at the outset has it been possible to induce any substantial lack of balance between fixation and evolution. Apparently in most circumstances the rate of production of the hydrogen acceptor governs the rate of carboxylation and this in turn limits the regeneration of the hydrogen acceptor and hence the rate of oxygen production. Against this background the regulatory effect of orthophosphate assumes added importance, particularly since its effects become apparent at less than 0.1 mM (Cockburn et al. 1967a,b,c). It has been reported by Preiss, Ghosh \& Wittkop (1965) that ADP-glucose pyrophosphorylase is inhibited by orthophosphate and activated by 3-phosphoglycerate and fructose 1,6-diphosphate. Although the present results almost certainly have no direct bearing on this system it seems clear that 
there might be a considerable regulatory interaction between the Calvin cycle and those reactions concerned in the synthesis of photosynthetic end products such as starch and sucrose.

This work was supported by the Science Research Council and, in part, by the University of London (Central Research Fund). The development of the oxygen electrode system was financed by the Royal Society. We are grateful to Dr R. Hill and Professor C. P. Whittingham for their continuing advice and criticism.

\section{REFERENCES}

Anderson, L., Worthen, L. E. \& Fuller, R. C. (1967). Plant Physiol. 42, S-48.

Arnon, D. I. (1949). Plant Physiol. 24, 1.

Baldry, C. W., Bucke, C. \& Walker, D. A. (1966a). Biochim. biophys. Acta, 126, 207.

Baldry, C. W., Walker, D. A. \& Bucke, C. (1966b). Biochem. J. 101, 642.

Bamberger, E. S. \& Gibbs, M. (1963). Plant Physiol. 38, x.

Bassham, J. A. \& Calvin, M. (1957). The Path of Carbon in Photosynthesis, pp. 56-70. Englewood Cliffs, N. J.: Prentice-Hall Inc.

Bucke, C., Walker, D. A. \& Baldry, C. W. (1966). Biochem. J. 101, 636.

Cockburn, W., Baldry, C. W. \& Walker, D. A. (1967a). Biochim. biophys. Acta, 131, 594.

Cockburn, W., Baldry, C. W. \& Walker, D. A. (1967b). Biochim. biophys. Acta, 143, 606.
Cockburn, W., Baldry, C. W. \& Walker, D. A. (1967c). Biochim. biophys. Acta, 143, 614.

Gibbs, M. (1967). Annu. Rev. Biochem. 86, 757.

Gibbs, M. \& Bamberger, E. S. (1962). Plant Physiol. 37, lxiii.

Gibbs, M., Bamberger, E. S., Ellyard, P. W. \& Everson, R. G. (1965). Proc. NATO Inst. Advanced Study, Biochemistry of the Chloroplast, Aberystwyth, vol. 2, p. 3. London: Academic Press (Inc.) Ltd.

Good, N. E., Winget, G. D., Winter, W., Connolly, T. N., Izawa, S. \& Singh, R. M. M. (1966). Biochemistry, 5, 467.

Heber, U. W. (1965). Proc. NATO Inst. Advanced Study, Biochemistry of the Chloroplast, Aberystwyth, vol. 2, p. 71. London: Academic Press (Inc.) Ltd.

Jensen, R. G. \& Bassham, J. A. (1966). Proc. nat. Acad. Sci., Wash., 56, 1095.

Kalberer, P. B., Buchanan, B. B. \& Arnon, D. I. (1967). Proc. nat. Acad. Sci., Wash., 57, 1542.

Paulsen, J. M. \& Lane, M. D. (1966). Biochemistry, 5, 2350.

Preiss, J., Ghosh, H. P. \& Wittkop, J. (1965). Proc. NATO Inst. Advanced Study, Biochemistry of the Chloroplast, Aberystwyth, vol. 2, p. 131. London: Academic Press (Inc.) Ltd.

Walker, D. A. (1965a). Plant Physiol. 40, 1157.

Walker, D. A. (1965b). Proc. NATO Inst. Advanced Study, Biochemistry of the Chloroplast, Aberystwyth, vol. 2, p. 53. London: Academic Press (Inc.) Ltd.

Walker, D. A., Cockburn, W. \& Baldry, C. W. (1967). Nature, Lond., 216, 597.

Walker, D. A. \& Hill, R. (1967). Biochim. biophys. Acta, 131, 330.

Weissbach, A., Horecker, B. L. \& Hurwitz, J. (1956). J. biol. Chem. 218, 715. 